\title{
Scoliosis in myelomeningocele: epidemiology, management, and functional outcome
}

\author{
Nishit Mummareddy, BA, Michael C. Dewan, MD, Michael R. Mercier, BA, Robert P. Naftel, MD, \\ John C. Wellons III, MD, MSPH, and Christopher M. Bonfield, MD \\ Department of Neurological Surgery, Vanderbilt University Medical Center, Nashville, Tennessee
}

\begin{abstract}
OBJECTIVE The authors aimed to provide an updated and consolidated report on the epidemiology, management, and functional outcome of cases of myelomeningocele (MMC) in patients with scoliosis.

METHODS A comprehensive literature search was performed using MEDLINE, Embase, Google Scholar, and the Cochrane Database of Systematic Reviews on cases of MMC in patients with scoliosis between 1980 and 2016. The initial search yielded 670 reports. After removing duplicates and applying inclusion criteria, we included 32 full-text original articles in this study.

RESULTS Pooled statistical analysis of the included articles revealed the prevalence of scoliosis in MMC patients to be $53 \%(95 \% \mathrm{Cl} 0.42-0.64)$. Slightly more females (56\%) are affected with both MMC and scoliosis than males. Motor level appears to be a significant predictor of prevalence, but not severity, of scoliosis in MMC patients. Treatment options for these patients include tethered cord release (TCR) and fusion surgeries. Curvature improvement and stabilization after TCR may be limited to patients with milder $\left(<50^{\circ}\right)$ curves. Meanwhile, more aggressive fusion procedures such as a combined anterior-posterior approach may result in more favorable long-term scoliosis correction, albeit with greater complication rates. Quality of life metrics including ambulatory status and sitting stability are influenced by motor level of the lesion as well as the degree of the scoliosis curvature.
\end{abstract}

CONCLUSIONS Scoliosis is among the most common and challenging comorbidities from which patients with MMC suffer. Although important epidemiological and management trends are evident, larger, prospective studies are needed to discover ways to more accurately counsel and more optimally treat these patients.

https://thejns.org/doi/abs/10.3171/2017.2.PEDS16641

KEY WORDS epidemiology; myelomeningocele; neural tube defect; scoliosis; spina bifida; treatment; spine

$\mathrm{M}$ YELOMENINGOCELE (MMC), characterized as a protruding sac of both the meninges and spinal cord, is the most frequent manifestation of neural tube defects, affecting nearly 1500 newborns in the United States annually and 0.2-6.5 newborns from every 1000 births globally. ${ }^{4,13,27,30}$ One of the most common and most severe skeletal complications of MMC is scoliosis, ${ }^{45}$ which can result in respiratory compromise, decreased mobility, skin breakdown, sitting and ambulation issues, and worsening of neurological symptoms. ${ }^{11,42}$ Thus, an understanding of the effects of scoliosis on patients with MMC is particularly important.

A thorough review on the intersection between MMC and scoliosis has not been recently conducted. Therefore, we aimed to provide an updated and consolidated report on the epidemiological observations and clinical outcomes on this topic. In this study, we aggregated data addressing the following questions, which were formulated both from clinical curiosity and literature shortcomings: 1) What is the prevalence of scoliosis in MMC patients? 2) Does a sex predilection exist for scoliosis in MMC? 3) How does motor level correlate with presence and degree of scoliosis? 4) What scoliosis treatment options are most commonly employed for patients with MMC, and what outcomes can be expected? 5) What is the quality of life in MMC patients with scoliosis?

\section{Methods}

A comprehensive literature search was performed using MEDLINE, Embase, Google Scholar, and the Cochrane Database of Systematic Reviews in May of 2016.

ABBREVIATIONS MeSH = Medical Subject Headings; MMC = myelomeningocele; PRISMA = Preferred Reporting Items for Systematic Reviews and Meta-Analyses; TCR $=$ tethered cord release.

SUBMITTED November 21, 2016. ACCEPTED February 1, 2017.

INCLUDE WHEN CITING Published online April 28, 2017; DOI: 10.3171/2017.2.PEDS16641. 
To achieve an encompassing and focused literature search, relevant Medical Subject Headings (MeSH) terms were divided into 2 groups: Group 1 (terms associated with MMC) and Group 2 (terms associated with scoliosis). Group 1 consisted of the following terms: "myelomeningocele," "meningomyelocele," "spina bifida," "spina bifida cystica," "tethered spinal cord," and "neural tube defect." Group 2 consisted of the following: "scoliosis" and "neuromuscular scoliosis." Relevant combinations of the MeSH terms in the groups were searched. The articles from these initial searches were screened so that we included only original English-language full-length articles published between 1980 and 2016. Review papers and any original publications with a sample of fewer than 25 patients were excluded. The articles were further screened to ensure that they provided data relevant to one or more of the aforementioned questions. Reports were excluded if they did not provide data relevant to the questions or if there was no clear delineation of patients with both MMC and scoliosis. The remaining articles were included in this systematic review. Within these included articles, patients were excluded if they had a form of spina bifida other than MMC. Furthermore, patients were excluded if they did not have scoliosis, even if they had other deformity (e.g. kyphosis). No other exclusion criteria were applied. The literature search was conducted according to Preferred Reporting Items for Systematic Reviews and Meta-Analyses (PRISMA) guidelines (Fig. 1).22

Two authors (N.M. and M.R.M.) independently reviewed the articles, extracted data, and discussed any disagreements before including or excluding any studies. Disputes were resolved by an arbiter (M.C.D.) before data were included in this manuscript. Level of evidence for each article was assigned according to Oxford Centre for Evidence-Based Medicine 2011 guidelines (http://www. cebm.net/ocebm-levels-of-evidence/). Significant findings from the review are shown in Figs. 2 and 3 and Tables 1-3.

For data analysis, no strict definition of scoliosis was applied while evaluating the patients in the articles owing to a lack of information about each patient's degree of curvature. As a result, we accepted the articles' definition of scoliosis. Many of the articles determined scoliosis based on the Cobb angle identified on radiographs. However, some of the reports did not clearly define their methods of determining scoliosis. There were no instances of reverse lordosis being considered as scoliosis.

Data analysis was performed using Meta-Essentials (http://www.erim.eur.nl/research-support/meta-essentials/). Standard error of prevalence was calculated using the following formula: $(\mathrm{p} \times(1-\mathrm{p}) / \mathrm{n})^{\wedge} 0.5$, where $\mathrm{p}$ is the prevalence and $\mathrm{n}$ is the study sample size. A random effects model with $95 \%$ CIs was used to generate the forest plots.

\section{Results}

\section{Article Selection and Characteristics}

The initial literature search with different combinations of MeSH terms, as described in the Methods section, yielded a total of 670 reports. Once duplicate articles were removed and exclusion criteria applied, 32 reports

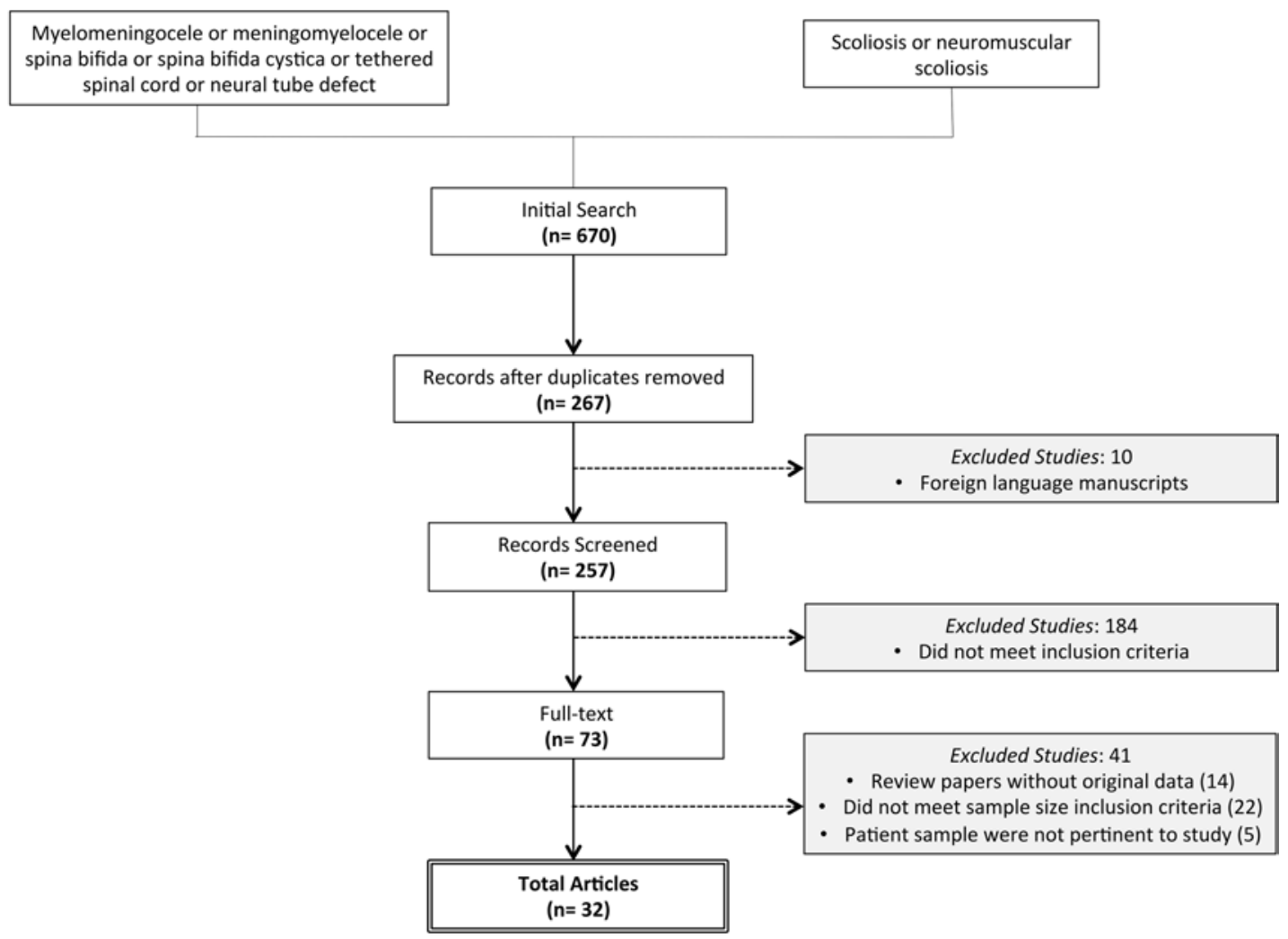

FIG. 1. PRISMA flow diagram for this study. 
remained and were included in this study (Fig. 1). Of these 32 articles, 7 were published in the 1980 s, 11 in the 1990 s, 5 in the 2000s, and 9 from 2010 until the present day. All but 3 of these articles were retrospective in design. The sample size of patients with both MMC and scoliosis in source articles ranged from 29 to 343 patients.

\section{Prevalence and Demographics of Scoliosis in MMC Patients}

A total of 15 articles provided data addressing the prevalence of scoliosis in patients with MMC (Fig. 2). Based on these studies, the percentage of MMC patients with scoliosis ranged widely from $23 \%$ to $88 \%{ }^{9,21,34,38}$ Pooled statistical analysis of the included studies revealed a prevalence rate of $52.8 \%$ (95\% CI 42\%-64\%). Díaz Llopis et al. and Reigel et al. conducted the 2 largest studies in terms of sample size with 1500 and 262 patients, respectively. Díaz Llopis et al. reported a prevalence rate of $23 \%$ in their population while Reigel et al. found a prevalence rate of $25 \%$. $^{9,31}$

Details about the sex ratio of patients with MMC and scoliosis were discussed in 17 studies (Fig. 3). In all but 3 studies, there were more females than males with MMCassociated scoliosis. Two studies found that males and females were affected equally, and only 1 article described males more commonly affected with both MMC and scoliosis at a ratio of 1.2:1. ${ }^{33,24,41}$ The largest sex disparity ratio of 2.2 females to 1 male was described in a 54-patient study by Banta in $1990 .^{2}$ Pooled statistical analysis of the included studies revealed that $56 \%$ (95\% CI 52\%-59\%) of the MMC patients with scoliosis were females.

\section{Effects of Motor Level on Scoliosis in MMC Patients}

One or more aspects of this topic were discussed in reports of 9 studies (Table 1). Five of these studies examined the percentage of MMC patients with scoliosis based on motor level, the lowest level of functionality in the spinal cord. ${ }^{5,23,31,35,42}$ Trivedi et al. found that $93 \%$ of MMC patients with thoracic lesions had scoliosis, whereas only $72 \%$ of patients with upper lumbar lesions (L1-3), $43 \%$ of patients with lower lumbar lesions (L4-5), and $8 \%$ of patients with sacral lesions did. ${ }^{42}$ Similarly, the remaining 4 studies also reported that lesions at the thoracic level resulted in the highest percentage of MMC patients with scoliosis. Most of these studies also found that sacral level lesions resulted in the least prevalence of scoliosis in MMC patients. However, a 71-patient prospective study by Bowman et al. reported in 2001 that upper lumbar lesions resulted in the lowest prevalence of scoliosis. ${ }^{5}$

One article published findings about the effects of motor level on the degree of scoliosis. ${ }^{1}$ This study found no association between motor level/MMC lesion level and the scoliosis curve. In addition, the authors reported the average preoperative scoliosis curves for thoracic, upper lumbar, and lower lumbar lesions to be $74^{\circ}, 75^{\circ}$, and $74^{\circ}$, respectively. After surgery, these curves were reduced to $41^{\circ}, 36^{\circ}$, and $40^{\circ}$, respectively..$^{1}$ One study in 2009 by Bowman et al. found that a majority (54\%) of the patients who experienced curve progression even after tethered cord release (TCR) had a thoracic motor level. ${ }^{6}$

In 1981, Kahanovitz and Duncan reported data regarding the effects of motor level on sitting stability and ambulation. They found that 55\% of MMC patients with scoliosis who were unbalanced sitters had a motor level of T-12. Furthermore, $100 \%$ of MMC and scoliosis patients with L-5 or S-1 motor levels were ambulatory. However, only $35 \%$ of the patients with a motor level above L-5 were ambulatory. ${ }^{14}$ Future research looking at MMC populations without scoliosis needs to be performed to establish an accurate comparison of motor level and ambulatory status. In addition, Samuelsson and Skoog found that an increasing scoliosis curve resulted in worse ambulatory outcomes. ${ }^{35}$

\section{Management of MMC Patients With Scoliosis}

Eighteen articles discussed one or more aspects of this topic, most of which addressed various surgical treatment

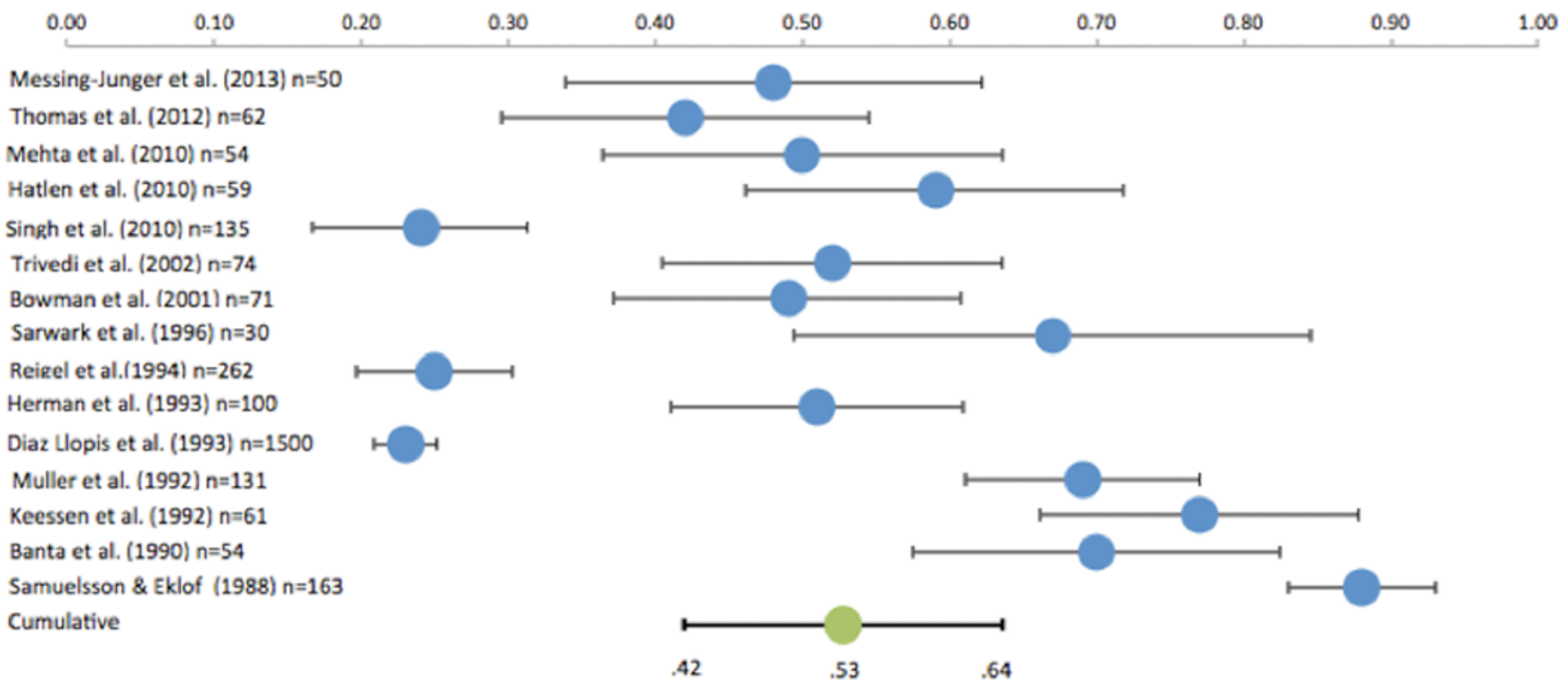

FIG. 2. Forest plot showing that $53 \%$ of patients with MMC also suffer from scoliosis. Figure is available in color online only. 


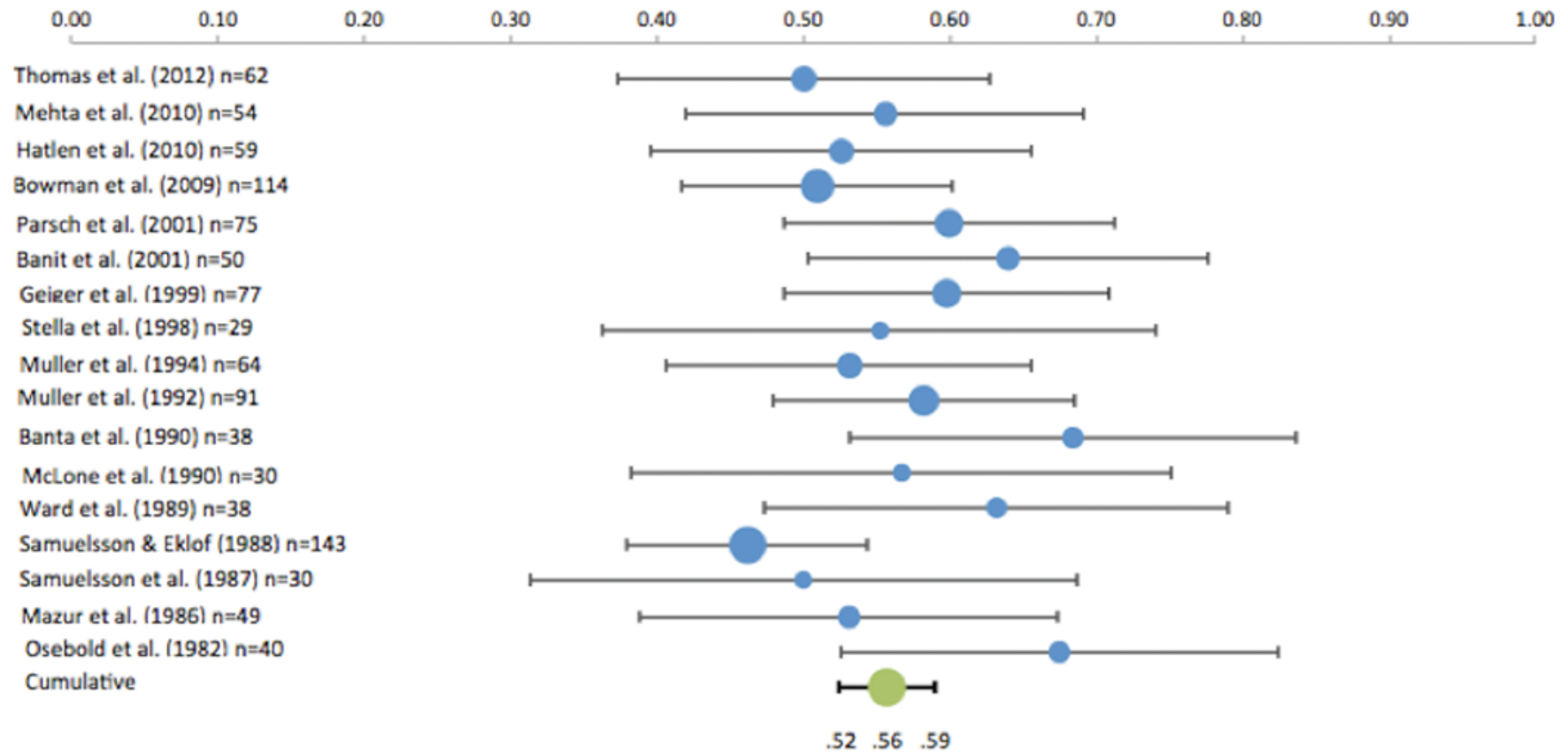

FIG. 3. Forest plot showing percentage of MMC patients with scoliosis who were female. More female than male patients tend to have both MMC and scoliosis. Figure is available in color online only.

options for these patients. Generally, the surgical approaches can be divided into one of two entities: TCR and instrumentation/fusion for scoliosis correction.

Seven studies examined the effects of TCR on MMC patients with scoliosis. Several reports found modest to moderate improvement in various symptoms after TCR. ${ }^{5,6,36}$ Sarwark and colleagues noted that $15 \%$ of their patients had improvement in scoliosis, while 60\% had stable curvature and $25 \%$ had curve progression during the 1-year follow-up after TCR. ${ }^{36}$ In a retrospective study

TABLE 1. Effects of motor level on scoliosis in MMC patients

\begin{tabular}{|c|c|c|c|c|}
\hline Authors \& Year & $\begin{array}{l}\text { Study } \\
\text { Design }\end{array}$ & $\begin{array}{l}\text { No. of } \\
\text { Patients }\end{array}$ & Findings & $\begin{array}{l}\text { Level of } \\
\text { Evidence }\end{array}$ \\
\hline Bowman et al., 2009 & Prospect & 114 & $54 \%$ of the patients $\mathrm{w} /$ progressive scoliosis after TCR had function at the thoracic level. & $3 b$ \\
\hline Trivedi et al., 2002 & Retro & 74 & $\begin{array}{l}93 \% \text { of patients w/ thoracic lesions had scoliosis. } 72 \% \text { w/ lumbar lesions had scoliosis. } \\
43 \% \text { of patients w/ lower lumbar lesions had scoliosis. } 8 \% \text { of patients w/ sacral lesions } \\
\text { had scoliosis. }\end{array}$ & 4 \\
\hline Banit et al., 2001 & Retro & 50 & $\begin{array}{l}\text { Mean scoliotic curve for thoracic, upper lumbar (L1-3), \& lower lumbar (L4-5) lesions were } \\
74^{\circ}, 75^{\circ}, \& 74^{\circ} \text {, respectively, preop \& } 41^{\circ}, 36^{\circ}, \& 40^{\circ} \text { postop. }\end{array}$ & 4 \\
\hline Bowman et al., 2001 & Prospect & 71 & $\begin{array}{l}51 \% \text { of scoliosis patients had a thoracic motor level, } 6 \% \text { an upper lumbar level, } 29 \% \text { a } \\
\text { lower lumbar level, \& } 11 \% \text { a sacral level. }\end{array}$ & $3 b$ \\
\hline Müller et al., 1994 & Retro & 64 & Level of lesion was not significantly associated w/ change in scoliosis angle. & 4 \\
\hline Reigel et al., 1994 & Retro & 262 & $\begin{array}{l}77 \% \text { of patients w/ thoracic lesions had scoliosis. } 40 \% \mathrm{w} / \text { lumbar lesions had scoliosis. } \\
13 \% \mathrm{w} / \text { lower lumbar lesions had scoliosis. } 3 \% \mathrm{w} / \text { sacral lesions had scoliosis. }\end{array}$ & 4 \\
\hline Müller et al., 1992 & Retro & 131 & $\begin{array}{l}94 \% \text { of patients w/ thoracic lesions had scoliosis. } 75 \% \text { w/ lumbar lesions had scoliosis. } \\
50 \% \text { w/ lower lumbar lesions had scoliosis. } 20 \% \text { w/ sacral lesions had scoliosis. Signifi- } \\
\text { cant correlation existed btwn functional level \& scoliosis prevalence. }\end{array}$ & 4 \\
\hline $\begin{array}{l}\text { Samuelsson \& Skoog, } \\
\quad 1988\end{array}$ & Retro & 163 & $\begin{array}{l}100 \% \text { of patients w/ thoracic lesions had scoliosis. } 95 \% \text { w/ L1-2 lesions had scoliosis. } \\
90 \% \mathrm{w} / \mathrm{L}-3 \text { lesions had scoliosis. } 85 \% \mathrm{w} / \mathrm{L}-4 \text { lesion had scoliosis. } 88 \% \mathrm{w} / \mathrm{L}-5 \text { lesion } \\
\text { had scoliosis. } 72 \% \mathrm{w} / \text { sacral lesions had scoliosis. Increasing scoliosis curve resulted in } \\
\text { worse ambulatory outcomes. }\end{array}$ & 4 \\
\hline $\begin{array}{l}\text { Kahanovitz et al., } \\
1981\end{array}$ & Retro & 39 & $\begin{array}{l}55 \% \text { of the patients who were unbalanced sitters had a T-12 motor level. } 100 \% \text { of L-5 \& } \\
\text { S-1 patients were ambulators. } 35 \% \text { of patients w/ neurological levels above L-5 were } \\
\text { ambulators. }\end{array}$ & 4 \\
\hline
\end{tabular}

Prospect $=$ prospective; retro $=$ retrospective 
TABLE 2. Surgical treatment outcomes and complications in MMC patients with scoliosis

\begin{tabular}{|c|c|c|c|c|c|}
\hline $\begin{array}{l}\text { Authors } \\
\& \text { Year }\end{array}$ & $\begin{array}{l}\text { Study } \\
\text { Design }\end{array}$ & $\begin{array}{l}\text { No. of } \\
\text { Patients }\end{array}$ & Treatment & Findings & $\begin{array}{l}\text { Level of } \\
\text { Evidence }\end{array}$ \\
\hline $\begin{array}{l}\text { Mehta et al., } \\
2010\end{array}$ & Retro & 54 & Surgery (TCR) & $\begin{array}{l}\text { Retethering occurred in } 41 \% \text { of scoliosis patients but only } 22 \% \text { of } \\
\text { patients w/o scoliosis }(p=0.149) \text {; furthermore, retethering occurred } \\
\text { earlier than in patients w/o scoliosis. }\end{array}$ & 4 \\
\hline $\begin{array}{l}\text { Hatlen et al., } \\
2010\end{array}$ & Retro & 59 & Surgery (spinal fusion) & $\begin{array}{l}\text { Complications, arising in more than half the cases, included infection } \\
\& \text { respiratory failure. } 12 \text { patients required a } 2 \text { nd op due to infection. } \\
\text { No correlation btwn MMC lesion \& complication rate. }\end{array}$ & 4 \\
\hline $\begin{array}{l}\text { Cahill et al., } \\
\qquad 2010\end{array}$ & Retro & 84 & Surgery & $18 \%$ experienced postop infection. & 4 \\
\hline $\begin{array}{r}\text { Bowman et } \\
\text { al., } 2009\end{array}$ & Prospect & 114 & Surgery (TCR) & $\begin{array}{l}\text { Scoliosis progressed after TCR in } 52 \% .70 \% \text { of patients showed } \\
\text { improved LE muscle strength. Gait was also improved. Spasticity } \\
\text { improved in } 67 \% \text {. } 64 \% \text { showed improvement in bladder function. } \\
\text { Complications of the op included CSF leak (6\%), infection (10\%), \& } \\
\text { neurological deterioration (4\%). }\end{array}$ & $3 b$ \\
\hline $\begin{array}{l}\text { Parsch et al., } \\
2001\end{array}$ & Retro & 54 & $\begin{array}{l}\text { Surgery } \\
\text { (Group I: PF/instrumenta- } \\
\text { tion; Group II: AF/no } \\
\text { instrumentation combined } \\
\text { w/ PF/instrumentation; } \\
\text { Group III: CF/instrumenta- } \\
\text { tion) }\end{array}$ & $\begin{array}{l}\text { Cobb angle for Group I changed from a mean of } 79^{\circ} \text { preop to } 35^{\circ} \\
\text { postop to } 45^{\circ} \text { at } 3.5 \text {-yr follow-up. } \\
\text { Cobb angle for Group II changed from mean of } 97^{\circ} \text { preop to } 52^{\circ} \text { post- } \\
\text { op to } 61^{\circ} \text { at } 3.4 \text {-yr follow-up. Cobb angle for Group III changed from } \\
\text { mean of } 92^{\circ} \text { preoperative to } 38^{\circ} \text { postop to } 40^{\circ} \text { at } 2.5 \text {-yr follow-up. } \\
\text { Overall hardware complication rate was } 30 \% \text {; these rates were not } \\
\text { significantly different btwn groups. Patients w/ thoracic lesion had a } \\
\text { greater loss of correction than patients w/ lumbar lesion }(p<0.06) \text {. }\end{array}$ & 4 \\
\hline $\begin{array}{l}\text { Banit et al., } \\
\quad 2001\end{array}$ & Retro & 50 & Surgery (PF) & $\begin{array}{l}\text { Cobb angle changed from a mean of } 75^{\circ} \text { preop to } 39^{\circ} \text { postop to } 45^{\circ} \\
\text { at follow-up. } 48 \% \text { had complications (infection, pseudomeningo- } \\
\text { cele, \& UTI). }\end{array}$ & 4 \\
\hline $\begin{array}{r}\text { Bowman et } \\
\text { al., } 2001\end{array}$ & Prospect & 71 & Surgery (TCR) & $\begin{array}{l}10 \text { patients developed symptomatic tethering involving scoliosis; } 50 \% \\
\text { of these patients had improvement in symptoms \& } 50 \% \text { became } \\
\text { stable. } 43 \% \text { of these patients eventually required spinal fusion ops, } \\
\text { the majority of which were at the thoracic level. }\end{array}$ & $3 b$ \\
\hline $\begin{array}{l}\text { Geiger et al., } \\
1999\end{array}$ & Prospect & 77 & $\begin{array}{l}\text { Surgery } \\
\text { (Group I: anterior correction } \\
\text { \& Harrington rods; Group } \\
\text { II: posterior instrumenta- } \\
\text { tion; Group III: an- } \\
\text { terior release \& posterior } \\
\text { instrumentation; Group } \\
\text { IV: anterior \& posterior } \\
\text { instrumentation) }\end{array}$ & 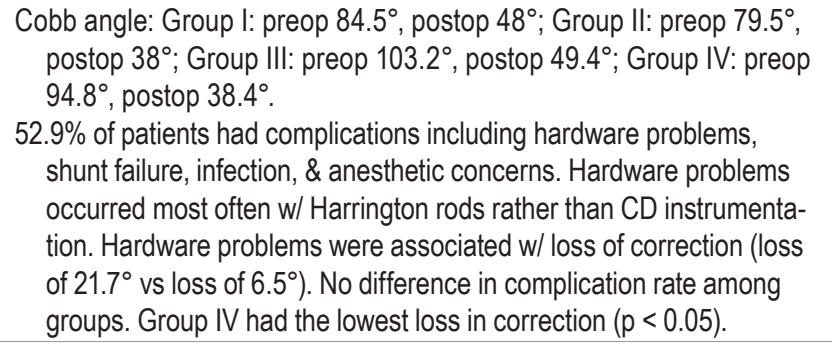 & $3 b$ \\
\hline $\begin{array}{l}\text { Stella et al., } \\
\quad 1998\end{array}$ & Retro & 29 & Surgery (7 PF, 3 AF, 19 CF) & $\begin{array}{l}\text { Posterior fusion resulted in scoliosis curve correction of } 22 \% \text { in tho- } \\
\text { racic \& thoracolumbar curves \& } 32 \% \text { in lumbar curves. Anterior fu- } \\
\text { sion resulted in scoliosis curve correction of } 43 \% \text { in lumbar curves. } \\
\text { Combined fusion resulted in scoliosis curve correction of } 55 \% \text { in } \\
\text { thoracic \& thoracolumbar curves \& } 61 \% \text { in lumbar curves. Overall, } \\
\text { ops resulted in } 47 \% \text { mean correction in thoracic \& thoracolumbar } \\
\text { curves \& } 50 \% \text { mean correction in lumbar curves. Complications of } \\
\text { ops included infection, bed sores, \& pneumothorax. }\end{array}$ & 4 \\
\hline $\begin{array}{l}\text { Sarwark et } \\
\text { al., } 1996\end{array}$ & Retro & 30 & Surgery (TCR) & $\begin{array}{l}\text { First-yr follow-up results showed } 15 \% \text { of patients w/ MMC \& scoliosis } \\
\text { improved, } 60 \% \text { were stable, \& } 25 \% \text { had curve progression. }\end{array}$ & 4 \\
\hline $\begin{array}{l}\text { Reigel et al., } \\
\quad 1994\end{array}$ & Retro & 262 & Surgery (TCR) & $\begin{array}{l}25 \% \text { of patients developed significant scoliosis }\left(>30^{\circ}\right) \text { after TCR. } \\
\text { Highest prevalence }(77 \%) \text { of scoliosis occurred in thoracic lesions. } \\
\text { Only for thoracic group did progression of scoliosis continue after } \\
\text { TCR. }\end{array}$ & 4 \\
\hline $\begin{array}{l}\text { Herman et } \\
\text { al., } 1993\end{array}$ & Retro & 100 & Surgery (TCR) & $\begin{array}{l}\text { Correction in the scoliosis curve by at least } 7^{\circ} \text { occurred in } 51 \% \text { of the } \\
\text { patients. } 10 \% \text { had progression of the curve at } 1 \text {-yr follow-up. } 63 \% \\
\text { improved or were stable in the follow-up exams after } 1 \text { year. CSF } \\
\text { leak \& infection were the most common complications. }\end{array}$ & 4 \\
\hline
\end{tabular}


» CONTINUED FROM PAGE 103

TABLE 2. Surgical treatment outcomes and complications in MMC patients with scoliosis

\begin{tabular}{|c|c|c|c|c|c|}
\hline $\begin{array}{l}\text { Authors } \\
\& \text { Year }\end{array}$ & $\begin{array}{l}\text { Study } \\
\text { Design }\end{array}$ & $\begin{array}{c}\text { No. of } \\
\text { Patients }\end{array}$ & Treatment & Findings & $\begin{array}{l}\text { Level of } \\
\text { Evidence }\end{array}$ \\
\hline $\begin{array}{r}\text { Keessen et } \\
\text { al., } 1992\end{array}$ & Retro & 48 & $\begin{array}{l}\text { Surgery }(P F n=15, A F n= \\
8, \text { circumferential fusion } \\
n=25)\end{array}$ & $\begin{array}{l}\text { AF: preop } 88.8^{\circ} \text {, postop } 37.1 \text {; PF: preop } 71.2^{\circ} \text {, postop } 45.7^{\circ} \text {; circum- } \\
\text { ferential: preop } 84.6^{\circ} \text {, postop } 31.1^{\circ} \text {. } \\
\text { Correction was } 45 \% \text { for thoracolumbar, } 20 \% \text { for thoracic, \& } 30 \% \text { for } \\
\text { lumbar. Complications included infection, instrumentation related, } \\
\text { skin issues, \& neurological. }\end{array}$ & 4 \\
\hline Banta, 1990 & Retro & 54 & Surgery (CF) & $\begin{array}{l}\text { Pre-op: } 73 \text { Post-op: } 34 \\
4 \text { patients needed revision surgery. } 47 \% \text { of patients had complications } \\
\text { w/ the procedures. The } 2 \text { most common were infection \& pseudar- } \\
\text { throsis. }\end{array}$ & 4 \\
\hline $\begin{array}{l}\text { McLone et } \\
\text { al., } 1990\end{array}$ & Retro & 30 & Surgery (TCR) & $\begin{array}{l}\text { At } 1 \text {-yr follow-up, } 83 \% \text { of patients } w / \text { curves }>50^{\circ} \text { progressed \& } \\
\text { required fusion. } 96 \% \text { w } / \text { curves }<50^{\circ} \text { improver or were stable. } \\
\text { However, at last follow-up, } 37 \% \text { progressed, } 21 \% \text { improved, \& } 42 \% \\
\text { remained the same. }\end{array}$ & 4 \\
\hline $\begin{array}{l}\text { Ward et al., } \\
1989\end{array}$ & Retro & 38 & $\begin{array}{l}\text { Surgery } \\
\text { (Group A: 1-stage AF or PF } \\
\text { w/ or w/o instrumentation; } \\
\text { Group B: anterior inter- } \\
\text { body fusion w/ posterior } \\
\text { fusion using Harrington } \\
\text { rod; Group C: anterior } \\
\text { interbody w/ Dwyer \& } \\
\text { posterior fusion using } \\
\text { Harrington; Group D: } \\
\text { anterior interbody fusion } \\
\text { w/ posterior fusion using } \\
\text { Luque; Group E: anterior } \\
\text { interbody w/ Dwyer \& } \\
\text { posterior fusion w/ Luque) }\end{array}$ & $\begin{array}{l}\text { Initial fusion success rates for the } 5 \text { groups were } 50 \%, 100 \%, 92 \% \text {, } \\
83 \%, \& 100 \% \text {, respectively. } 16 \% \text { of patients needed to have a } 2 \text { nd } \\
\text { procedure. } \\
\text { Group A: preop 69, postop 64; Group B: preop 81, postop 35; Group } \\
\text { C: preop } 77 \text {, postop } 43 ; \text { Group D: preop } 57 \text {, postop } 28 \text {; Group E: } \\
\text { preop } 62 \text {, postop } 18 \text {. } \\
\text { Group B \& E improvements were significantly better than A. } 58 \% \text { of } \\
\text { patients had complications. }\end{array}$ & 4 \\
\hline $\begin{array}{l}\text { Mazur et al., } \\
1986\end{array}$ & Retro & 49 & $\begin{array}{l}\text { Surgery } \\
\text { (Group A: PF; Group B: AF; } \\
\quad \text { Group C: CF) }\end{array}$ & $\begin{array}{l}\text { Cobb angle for Group A changed from a mean of } 78^{\circ} \text { preop to } 41^{\circ} \\
\text { postop to } 53^{\circ} \text { at follow-up. } \\
\text { Cobb angle for Group B changed from a mean of } 61^{\circ} \text { preop to } 27^{\circ} \\
\text { postop to } 32^{\circ} \text { at follow-up. } \\
\text { Cobb angle for Group C changed from a mean of } 78^{\circ} \text { preop to } 33^{\circ} \text { po- } \\
\text { stop to } 45^{\circ} \text { at follow-up. No significant difference among groups in } \\
\text { terms of postop function. Infections only occurred during posterior } \\
\text { ops (Group A } 7 \% \text {; Group C } 11 \% \text { ). Pseudarthrosis rate was } 33 \% \text {, } \\
29 \%, \& 11 \% \text {, respectively. }\end{array}$ & 4 \\
\hline $\begin{array}{r}\text { Osebold et } \\
\text { al., } 1982\end{array}$ & Retro & 40 & $\begin{array}{l}\text { Surgery } \\
\text { (Group A: PF; Group B: PF } \\
\text { \& instrumentation; Group } \\
\text { C: PF \& instrumentation } \\
\text { along w/ AF; Group D: PF } \\
\text { \& instrumentation along } \\
\text { w/ AF \& instrumentation) }\end{array}$ & $\begin{array}{l}\text { Average correction at follow-up for scoliosis was } 6^{\circ}, 12^{\circ}, 52^{\circ}, \& 45^{\circ} \text {, } \\
\text { respectively, in Groups A-D. Pseudarthrosis occurred in } 67 \% \text {, } \\
46 \%, 100 \%, \& 23 \% \text {, \& infection in } 23 \%, 33 \%, 29 \%, \& 8 \% \text {. }\end{array}$ & 4 \\
\hline
\end{tabular}

$\mathrm{AF}=$ anterior fusion; $\mathrm{CD}=$ Cotrel-Dubousset; $\mathrm{CF}=$ combined fusion; $\mathrm{LE}=$ lower extremity; $\mathrm{PF}=$ posterior fusion; UTI = urinary tract infection.

in 1993, Herman et al. found that there was a correction of the scoliosis curve by at least $7^{\circ}$ in $51 \%$ of the patients postoperatively after TCR. This study also reported that $63 \%$ of the patients with at least a 1-year follow-up improved or demonstrated curve stability.12 McLone and colleagues examined curve reduction after surgery, based on the degree of scoliosis. Almost all patients (96\%) with a curve less than $50^{\circ}$ had curve improvement during the 12-month post-TCR follow-up period. However, they did note that curves in only $63 \%$ of the patients with initial curves less than $50^{\circ}$ were considered to be improved or stable at the last follow-up visit several years after the procedure. Meanwhile curves in $83 \%$ of the patients with a curve greater than $50^{\circ}$ progressed and required spinal fusion surgery. ${ }^{17}$ Retethering of the spinal cord occurred in $41 \%$ of patients with scoliosis as opposed to only $22 \%$ of 
TABLE 3. Effects of scoliosis on quality of life for myelomeningocele patients

\begin{tabular}{|c|c|c|c|c|}
\hline Authors \& Year & $\begin{array}{l}\text { Study } \\
\text { Design }\end{array}$ & $\begin{array}{l}\text { No. of } \\
\text { Patients }\end{array}$ & Findings & $\begin{array}{l}\text { Level of } \\
\text { Evidence }\end{array}$ \\
\hline Thomas et al., 2012 & Retro & 62 & Mean Hoffer score was 2.6. 16.4\% scored 1, 8.2\% scored 2, \& 75.4\% scored 3. & 4 \\
\hline Patel et al., 2011 & Retro & 32 & $\begin{array}{l}80 \% \text { of patients used wheelchairs for all mobility. } 20 \% \text { were household ambulators \& used } \\
\text { wheelchairs in the community. As magnitude of curve increased, there was an increase } \\
\text { in the percentage of seat w/ a pressure btwn } 38-70 \mathrm{~mm} \mathrm{Hg} \text {. }\end{array}$ & 4 \\
\hline Bowman et al., 2009 & Prospect & 114 & $\begin{array}{l}78 \% \text { showed improvement in gait, } 19 \% \text { remained stable, \& } 3 \% \text { got worse. All showed } \\
\text { postop improvement in pain. }\end{array}$ & $3 b$ \\
\hline Trivedi et al., 2002 & Retro & 74 & Significant association btwn scoliosis \& nonambulatory status. & 4 \\
\hline Stella et al., 1998 & Prospect & 29 & $\begin{array}{l}4 \text { patients w/o orthoses or crutches; } 2 \text { w/ orthoses \& w/o crutches; } 9 \text { w/ both. } 14 \text { dependent } \\
\text { on wheelchairs. }\end{array}$ & $3 b$ \\
\hline Sarwark et al., 1996 & Retro & 30 & $\begin{array}{l}\text { Sitting balance was improved after surgery. All patients w/ preop back pain had pain resolu- } \\
\text { tion. }\end{array}$ & 4 \\
\hline Mazur et al., 1986 & Retro & 49 & $\begin{array}{l}\text { Ability to ambulate deteriorated in } 27 \% \text { of Group A, } 57 \% \text { of Group B, \& } 67 \% \text { of Group C. } \\
73 \% \text { of Group A, } 43 \% \text { of Group C, \& } 33 \% \text { of Group C remained the same. } 67 \% \text { of Group } \\
\text { A \& } 70 \% \text { of Group C showed improved sitting ability. Group C showed most improvement } \\
\text { w/ sitting. In Groups A \& C, the majority of patients' back pain was unchanged. In Group } \\
\text { B, } 43 \% \text { improved, } 43 \% \text { remained the same. }\end{array}$ & 4 \\
\hline Osebold et al., 1982 & Retro & 40 & $57 \%$ of interviewed patients said major benefit was improved sitting stability. & 4 \\
\hline $\begin{array}{l}\text { Kahanovitz \& Duncan, } \\
\quad 1981\end{array}$ & Retro & 39 & $\begin{array}{l}\text { At final follow-up, } 20 \text { patients were nonambulators, } 9 \text { were balanced sitters, \& } 11 \text { were unbal- } \\
\text { anced sitters. The remaining } 19 \text { patients were ambulatory ( } 5 \text { household, } 14 \text { community). }\end{array}$ & 4 \\
\hline
\end{tabular}

patients without scoliosis in a study performed by Mehta et al. However, this difference was not significant $(\mathrm{p}=$ 0.149 ) In addition, the retethering occurred significantly earlier in patients with scoliosis than it did in patients without scoliosis $(\mathrm{p}=0.042) .{ }^{19}$

Spinal fusion for MMC patients with scoliosis was discussed in 11 studies. Several of these reports included comparisons of different spinal surgical approaches with one another. ${ }^{10,15,28,38}$ Parsch et al. examined the Cobb angle change in patients who underwent posterior instrumentation/fusion (Group 1), anterior fusion with posterior instrumentation/fusion (Group 2), and combined instrumentation/fusion (Group 3). The Cobb angle for patients in Group 1 changed from an average of $79^{\circ}$ preoperatively to $35^{\circ}$ postoperatively to $45^{\circ}$ at follow-up. In Group 2, the Cobb angle changed from $97^{\circ}$ preoperatively to $52^{\circ}$ postoperatively to $63^{\circ}$ at follow-up. In Group, 3 the Cobb angle changed from $92^{\circ}$ preoperatively to $38^{\circ}$ postoperatively to $40^{\circ}$ at last follow-up. ${ }^{28}$ Similar to these findings, Mazur et al. and Geiger et al. also published series in which they found a greater correction of the scoliosis curve when combined fusion was used as the surgical intervention. ${ }^{10,16}$

In terms of complication rate after posterior fusion surgery, one study found that $48 \%$ of patients had complications while another reported that $53 \%$ of their patients suffered complications such as hardware problems, shunt failures, infections, and pseudomeningoceles. ${ }^{1,10}$ In a study by Banit et al. with a $48 \%$ complication rate, no patient's treatment resulted in cardiopulmonary complications or death. However, $63 \%$ of the patients with complications required an additional surgery. ${ }^{1}$ Geiger and colleagues reported a complication rate of $53 \%$, but they found that there was no difference in the complication rates among the different surgical techniques. The 4 main groups of complications included hardware problems, shunt failures, infection, and anesthetic complications. ${ }^{10}$ Cahill and colleagues found that an alarming $18 \%$ of their population had infection after surgery. ${ }^{7}$ In addition, one study found no correlation between MMC level and complication rate, whereas another published data suggesting that postoperative infection only occurred in patients who had undergone posterior surgical approaches..$^{11,16}$

\section{Quality of Life in MMC Patients With Scoliosis}

Quality of life in terms of ambulatory status and sitting stability was discussed in 9 articles (Table 3). Thomas et al. reported a mean Hoffer score of 2.6 in a sample of 62 MMC and scoliosis patients, with $16.4 \%$ of the patients scoring a 1 (ambulatory with prosthetic device), 8.2\% scoring a 2 (partially ambulatory with prosthetic device), and $75.4 \%$ scoring a 3 (wheelchair bound). The median motor level was L-1, with a range from T-4 to $\mathrm{S}-1 .{ }^{41}$ In the cohort of 32 patients that Patel et al. studied, $80 \%$ of the patients used wheelchairs for all mobility, while $20 \%$ of the patients were ambulatory in the household but used wheelchairs in the community. ${ }^{29}$ As would be expected, one study found that nonambulatory patients were more likely to develop scoliosis $(\mathrm{p}<0.001) .{ }^{42}$ This suggests that nonambulatory status plays a causative role in the development of scoliosis, not vice versa. However, Kahanovitz and Duncan reported a much more balanced ratio of patients who were nonambulatory $(51 \%)$ and patients who were ambulatory $(49 \%) .{ }^{14}$

Sitting stability is a valid quality of life metric in children with neuromuscular pathology as abnormal balance can cause discomfort and skin breakdown. ${ }^{3}$ In patients with MMC-related scoliosis, Patel et al. noted that an increase in the magnitude of the scoliosis curve resulted in an increased percentage of the contact area between the 
buttocks and the wheelchair with a pressure between 38 and $70 \mathrm{~mm} \mathrm{Hg} .{ }^{29}$ Three studies reported improvement in sitting stability for their patients who underwent a surgical procedure. ${ }^{16,26,36}$

\section{Discussion}

In the present study, we screened a total of 670 reports and ultimately included 32 full-length articles that discuss scoliosis in MMC patients. Although the prevalence of MMC in the population has declined since the addition of folate into a regular diet, a significant population still suffers from MMC and its severe bony complication, scoliosis. ${ }^{13} \mathrm{~A}$ thorough understanding of this topic, therefore, is essential when counseling and managing these patients. To our knowledge this study is the most comprehensive and up-to-date systematic review examining the most important clinical questions surrounding MMC patients suffering from scoliosis. We have sought to address the epidemiology and management outcomes in these patients in a concise and straightforward report.

From our literature search and analysis, several significant patterns were identified and we elaborate on them below. More female MMC patients had scoliosis than male MMC patients. ${ }^{1,43}$ Whether this increased female predilection is due to an underlying pathophysiological process or whether it is simply due to higher rates of females suffering from MMC remains uncertain. Sawin and colleagues published a report highlighting the demographics of the patients included in the National Spina Bifida Patient Registry, a multi-institution project with over 1500 patients with MMC. They reported that more females (51.6\%) suffered from MMC than males. ${ }^{37}$ Given that the higher percentage of MMC patients was female (51.6\%) and the higher percentage of patients having both MMC and scoliosis was also female (56\%), it seems likely that the increased prevalence of females suffering from MMC and scoliosis is a result of more females suffering from MMC than males. However, there is a slight difference in the percentages of the 2 groups. Whether this difference is significant or due to study limitation remains to be determined.

This bias toward females being more likely to have scoliosis with MMC is particularly important given that some of these females could get pregnant during their lives. A recent review on the effects of scoliosis on pregnancy in females suffering from adolescent idiopathic scoliosis found that these females tended to have higher rates of nulliparity and increased back pain prepartum (M. Dewan et al., unpublished data). Future studies evaluating the effects of scoliosis on pregnancy in MMC patients may provide useful answers in an otherwise unstudied area of the literature.

Several of the articles we reviewed indicated that the MMC lesion level/motor level was highly correlated with the prevalence of scoliosis in those patients but not with the severity of the curve. . $^{1,5,24,24,31}$ Basically, the higher the lesion level, the more likely the patient is to get scoliosis. This finding could potentially be an important diagnostic tool to determine whether early treatment, or at minimum screening, for scoliosis is warranted. While this topic is still hotly debated, early detection of scoliosis has been associated with better results for the use of noninvasive brace therapy. ${ }^{8,18,40}$ Regardless of treatment approach, MMC patients might benefit from earlier treatment of their scoliosis or more frequent monitoring based on lesion level; clinicians should be cognizant of this observation.

The data presented here show that TCR for MMC patients with scoliosis has mixed results. Several studies reported that a significant number of patients had improvement in their scoliosis after TCR, whereas a sizeable subset had scoliosis progression even after TCR., ${ }^{6,12,36}$ However, several of the articles did present data that suggested certain populations of MMC patients with scoliosis could benefit more from TCR than others. McLone et al. found that $96 \%$ of their MMC patients with a scoliosis curve of less than $50^{\circ}$ either improved or were stable during the 1-year follow-up period..$^{17}$ Whether $50^{\circ}$ of scoliosis curvature is a strong predictor of TCR surgery success needs to be validated with larger studies. However, based on the available data, this factor could serve as a tool for clinicians to counsel patients. In addition, it must be noted that because these results were based on a short follow-up interval of 1 year, the true efficacy of this surgery can only be gleaned from an analysis of results over a longer follow-up period. In addition, Reigel and colleagues reported that progression of the scoliotic curve after TCR occurred solely in patients with thoracic lesions. ${ }^{31}$ These collective findings suggest that TCR is potentially beneficial to some but not all MMC patients with scoliosis, specifically patients with smaller scoliosis and curves and lower-level lesions.

Whether untethering of the spinal cord should be performed before fusion surgery, at the same time of fusion surgery, or not at all still remains to be determined. Mehta and colleagues found that patients who were treated with concomitant TCR and scoliosis corrective surgery had less retethering, lower prevalence of wound infection, shorter operative time, and fewer hospital days than patients who had a 2-staged surgery for untethering at one stage and then scoliosis correction at another. ${ }^{20}$ However, this study included patients with pathologies other than MMCs, including thickened filum terminale and lipomyelomeningocele. In addition, Mehta and colleagues looked primarily at patients with large curves $\left(>40^{\circ}\right)$ and patients who had curves that were progressing. Another study showed that an additional neurosurgical procedure at the time of scoliosis correction surgery did not increase the complication rate compared with that in patients who only underwent scoliosis correction surgery. ${ }^{25}$ These reports suggest that performing TCR and scoliosis correction simultaneously may carry benefits for patients without increasing the complication rate. However, selecting the correct patient for this combined approach is absolutely necessary to ensure the best care. Samdani et al. found that untethering prior to fusion may actually be unnecessary in patients with MMC who do not have clinical symptoms of tethered cord, even if tethering is radiologically demonstrated..$^{32}$ As a result, being asymptomatic may be a contraindication to combined TCR and scoliosis correction surgery. Since all of these studies were retrospective in nature with no controls, it is difficult to generate a concrete set of guidelines on this topic. Indeed, larger studies with a prospective design and objective end points are needed to provide a conclusive answer. 
In terms of treating MMC patients with scoliosis by implanting spinal instrumentation, we found that indications for spinal fusion were only defined in some of the articles we reviewed. Specific indications were rarely defined, as the most common indication for surgery was listed as paralytic scoliosis. ${ }^{1,16,25,28}$ In addition, Keessen and colleagues used progressive sitting imbalance, pain, and pressure sores as indications for surgery. ${ }^{15}$ Again, larger studies that track patients for long periods both before and after treatment will help clinicians determine the optimal surgical candidates in the future. Furthermore, none of the reviewed articles reported any concrete numerical cutoffs in terms of goals for scoliosis correction. The authors of the reviewed articles were attempting to reduce the scoliosis curve as much as possible for the longest period of time.

Multiple studies have indicated that combined anterior-posterior fusion results in greater correction than an anterior or posterior approach alone..$^{10,16,26,28}$ Despite the increase in the correction, the combined instrumentation/ fusion surgeries are usually more invasive, which seems to result in more complications. Although 2 studies indicated no significant difference in complication rates between the combined fusion group and other groups, these studies did not analyze important perioperative considerations such as length of hospitalization, blood loss, and recovery time..$^{10,28}$ Furthermore, one study reported no significant difference in function between the different surgical groups. ${ }^{16}$ The heterogeneous nature of scoliosis demands customized treatment modality to address the level of the lesion, degree of the curve, and functional capacity of each individual patient. Realistically, answers are likely to emerge not from randomized controlled trials but rather from larger cohort studies evaluating long-term outcomes and patientreported metrics.

Complication rates for spinal surgery in MMC patients with scoliosis were reported to be $48 \%$ by Banit et al. and $53 \%$ by Geiger et al. ${ }^{1,10}$ These rates are higher than the average complication rate of $22 \%$ for spinal surgeries in patients with idiopathic scoliosis published by Weiss and Goodall. ${ }^{44}$ However, variations in complication rates for idiopathic scoliosis patients ranged from $0 \%$ to $73 \%$. The higher complication rate seen in MMC patients could be due to a number of factors including larger scoliosis curves, more comorbidities, and a lower capacity for rehabilitation after surgery. ${ }^{1,10,44}$

\section{Study Limitations}

It is important for readers to be cautious while interpreting conclusions from this review because of several limitations. First, the majority of the articles reviewed in this study were retrospective in nature. Thus, the level of evidence was relatively low. Furthermore, each of the articles had unique methodologies and criteria for inclusion, which could result in similarities and/or differences that might not be completely accurate. For example, the definition of scoliosis was not standardized across the articles reviewed, mainly due to missing information about the topic in the original reports. Nonstandardized disease definition may result in variations more reflective of literature disparities rather than true biological or clinical variation. As a result, prospective, controlled cohort studies with strict inclusion criteria are necessary to garner more conclusive data.

\section{Conclusions}

Scoliosis is among the most common skeletal abnormalities from which MMC patients suffer, potentially leading to respiratory comprise and significant mobility limitations. Pooled statistical analysis demonstrated a prevalence of scoliosis in MMC patients of 53\%, with females being slightly more affected (56\%) than males. Motor level is a significant predictor of prevalence of scoliosis in MMC patients but not of severity. Treatment options for these patients include TCR and fusion surgeries; however, not all patients benefit from these procedures. One useful marker to predict the success of TCR release might be the degree of scoliosis curvature. Furthermore, combined anteriorposterior fusion surgeries may result in better long-term outcomes despite greater perioperative complications. In the future, larger prospective studies will be important to gain conclusive evidence regarding the optimal surgical treatment of patients with MMC and scoliosis.

\section{References}

1. Banit DM, Iwinski HJ Jr, Talwalkar V, Johnson M: Posterior spinal fusion in paralytic scoliosis and myelomeningocele. $\mathbf{J}$ Pediatr Orthop 21:117-125, 2001

2. Banta JV: Combined anterior and posterior fusion for spinal deformity in myelomeningocele. Spine (Phila Pa 1976) 15:946-952, 1990

3. Bartnicki B, Synder M, Kujawa J, Stańczak K, Sibiński M: Siting stability in skeletally mature patients with scoliosis and myelomeningocele. Ortop Traumatol Rehabil 14:383389, 2012

4. Bhide P, Sagoo GS, Moorthie S, Burton H, Kar A: Systematic review of birth prevalence of neural tube defects in India. Birth Defects Res A Clin Mol Teratol 97:437-443, 2013

5. Bowman RM, McLone DG, Grant JA, Tomita T, Ito JA: Spina bifida outcome: a 25-year prospective. Pediatr Neurosurg 34:114-120, 2001

6. Bowman RM, Mohan A, Ito J, Seibly JM, McLone DG: Tethered cord release: a long-term study in 114 patients. J Neurosurg Pediatr 3:181-187, 2009

7. Cahill PJ, Warnick DE, Lee MJ, Gaughan J, Vogel LE, Hammerberg KW, et al: Infection after spinal fusion for pediatric spinal deformity: thirty years of experience at a single institution. Spine (Phila Pa 1976) 35:1211-1217, 2010

8. Coillard C, Circo AB, Rivard CH: SpineCor treatment for juvenile idiopathic scoliosis: SOSORT award 2010 winner. Scoliosis 5:25, 2010

9. Díaz Llopis I, Bea Muñoz M, Martinez Agulló E, López Martinez A, García Aymerich V, Forner Valero JV: Ambulation in patients with myelomeningocele: a study of $1500 \mathrm{pa}-$ tients. Paraplegia 31:28-32, 1993

10. Geiger F, Parsch D, Carstens C: Complications of scoliosis surgery in children with myelomeningocele. Eur Spine J 8:22-26, 1999

11. Hatlen T, Song K, Shurtleff D, Duguay S: Contributory factors to postoperative spinal fusion complications for children with myelomeningocele. Spine (Phila Pa 1976) 35:12941299,2010

12. Herman JM, McLone DG, Storrs BB, Dauser RC: Analysis of 153 patients with myelomeningocele or spinal lipoma reoperated upon for a tethered cord. Presentation, management and outcome. Pediatr Neurosurg 19:243-249, 1993

13. Januschek E, Röhrig A, Kunze S, Fremerey C, Wiebe B, 
Messing-Jünger M: Myelomeningocele-a single institute analysis of the years 2007 to 2015. Childs Nerv Syst 32:1281-1287, 2016

14. Kahanovitz N, Duncan JW: The role of scoliosis and pelvic obliquity on functional disability in myelomeningocele. Spine (Phila Pa 1976) 6:494-497, 1981

15. Keessen W, van Ooy A, Pavlov P, Pruijs JE, Scheers MM, Slot G, et al: Treatment of spinal deformity in myelomeningocele: a retrospective study in four hospitals. Eur J Pediatr Surg 2 (Suppl 1):18-22, 1992

16. Mazur J, Menelaus MB, Dickens DR, Doig WG: Efficacy of surgical management for scoliosis in myelomeningocele: correction of deformity and alteration of functional status. J Pediatr Orthop 6:568-575, 1986

17. McLone DG, Herman JM, Gabrieli AP, Dias L: Tethered cord as a cause of scoliosis in children with a myelomeningocele. Pediatr Neurosurg 16:8-13, 1990-1991

18. Mehta S, Betz RR, Mulcahey MJ, McDonald C, Vogel LC, Anderson C: Effect of bracing on paralytic scoliosis secondary to spinal cord injury. J Spinal Cord Med 27 (Suppl 1):S88-S92, 2004

19. Mehta VA, Bettegowda C, Ahmadi SA, Berenberg P, Thomale UW, Haberl EJ, et al: Spinal cord tethering following myelomeningocele repair. J Neurosurg Pediatr 6:498-505, 2010

20. Mehta VA, Gottfried ON, McGirt MJ, Gokaslan ZL, Ahn ES, Jallo GI: Safety and efficacy of concurrent pediatric spinal cord untethering and deformity correction. J Spinal Disord Tech 24:401-405, 2011

21. Messing-Jünger M, Röhrig A: Primary and secondary management of the Chiari II malformation in children with myelomeningocele. Childs Nerv Syst 29:1553-1562, 2013

22. Moher D, Shamseer L, Clarke M, Ghersi D, Liberati A, Petticrew M, et al: Preferred reporting items for systematic review and meta-analysis protocols (PRISMA-P) 2015 statement. Syst Rev 4:1, 2015

23. Müller EB, Nordwall A: Prevalence of scoliosis in children with myelomeningocele in western Sweden. Spine (Phila Pa 1976) 17:1097-1102, 1992

24. Müller EB, Nordwall A, Odén A: Progression of scoliosis in children with myelomeningocele. Spine (Phila Pa 1976) 19:147-150, 1994

25. Murans G, Gustavsson B, Saraste H: One-stage major spine deformity correction surgery: comparison between groups with and without additional neurosurgical intervention, with more than 24 months of follow-up. Clinical article. J Neurosurg Spine 13:666-671, 2010

26. Osebold WR, Mayfield JK, Winter RB, Moe JH: Surgical treatment of paralytic scoliosis associated with myelomeningocele. J Bone Joint Surg Am 64:841-856, 1982

27. Parker SE, Mai CT, Canfield MA, Rickard R, Wang Y, Meyer RE, et al: Updated National Birth Prevalence estimates for selected birth defects in the United States, 2004-2006. Birth Defects Res A Clin Mol Teratol 88:1008-1016, 2010

28. Parsch D, Geiger F, Brocai DR, Lang RD, Carstens C: Surgical management of paralytic scoliosis in myelomeningocele. J Pediatr Orthop B 10:10-17, 2001

29. Patel J, Walker JL, Talwalkar VR, Iwinski HJ, Milbrandt TA: Correlation of spine deformity, lung function, and seat pressure in spina bifida. Clin Orthop Relat Res 469:1302-1307, 2011

30. Rabiu TB, Adeleye AO: Prevention of myelomeningocele: African perspectives. Childs Nerv Syst 29:1533-1540, 2013

31. Reigel DH, Tchernoukha K, Bazmi B, Kortyna R, Rotenstein D: Change in spinal curvature following release of tethered spinal cord associated with spina bifida. Pediatr Neurosurg 20:30-42, 1994

32. Samdani AFFA, Fine AL, Sagoo SS, Shah SC, Cahill PJ, Clements DH, et al: A patient with myelomeningocele: is un- tethering necessary prior to scoliosis correction? Neurosurg Focus 29(1):E8, 2010

33. Samuelsson L, Bergström K, Thuomas KA, Hemmingsson A, Wallensten R: MR imaging of syringohydromyelia and Chiari malformations in myelomeningocele patients with scoliosis. AJNR Am J Neuroradiol 8:539-546, 1987

34. Samuelsson L, Eklöf O: Scoliosis in myelomeningocele. Acta Orthop Scand 59:122-127, 1988

35. Samuelsson L, Skoog M: Ambulation in patients with myelomeningocele: a multivariate statistical analysis. J Pediatr Orthop 8:569-575, 1988

36. Sarwark JF, Weber DT, Gabrieli AP, McLone DG, Dias L: Tethered cord syndrome in low motor level children with myelomeningocele. Pediatr Neurosurg 25:295-301, 1996

37. Sawin KJ, Liu T, Ward E, Thibadeau J, Schechter MS, Soe MM, et al: The National Spina Bifida Patient Registry: profile of a large cohort of participants from the first 10 clinics. J Pediatr 166:444-450, 450.e1, 2015

38. Singh D, Rath GP, Dash HH, Bithal PK: Anesthetic concerns and perioperative complications in repair of myelomeningocele: a retrospective review of 135 cases. J Neurosurg Anesthesiol 22:11-15, 2010

39. Stella G, Ascani E, Cervellati S, Bettini N, Scarsi M, Vicini M, et al: Surgical treatment of scoliosis associated with myelomeningocele. Eur J Pediatr Surg 8 (Suppl 1):22-25, 1998

40. Sy N, Borysov M, Moramarco M, Nan XF, Weiss HR: Bracing scoliosis - state of the art (mini-review). Curr Pediatr $\operatorname{Rev} 12: 36-42,2016$

41. Thomas JG, Hwang SW, Blumberg TJ, Whitehead WE, Curry DJ, Luerssen TG, et al: Correlation between shunt series and scoliosis radiographs in children with myelomeningoceles. J Neurosurg Spine 17:410-414, 2012

42. Trivedi J, Thomson JD, Slakey JB, Banta JV, Jones PW: Clinical and radiographic predictors of scoliosis in patients with myelomeningocele. J Bone Joint Surg Am 84-A:1389-1394, 2002

43. Ward WT, Wenger DR, Roach JW: Surgical correction of myelomeningocele scoliosis: a critical appraisal of various spinal instrumentation systems. J Pediatr Orthop 9:262268, 1989

44. Weiss HR, Goodall D: Rate of complications in scoliosis surgery-a systematic review of the Pub Med literature. Scoliosis 3:9, 2008

45. Westcott MA, Dynes MC, Remer EM, Donaldson JS, Dias LS: Congenital and acquired orthopedic abnormalities in patients with myelomeningocele. Radiographics 12:1155-1173, 1992

\section{Disclosures}

The authors report no conflict of interest concerning the materials or methods used in this study or the findings specified in this paper.

\section{Author Contributions}

Conception and design: Dewan, Naftel, Wellons, Bonfield. Acquisition of data: Mummareddy, Mercier. Analysis and interpretation of data: Mummareddy, Dewan, Mercier, Bonfield. Drafting the article: Mummareddy, Dewan. Critically revising the article: Mummareddy, Dewan, Naftel, Wellons, Bonfield. Reviewed submitted version of manuscript: all authors. Approved the final version of the manuscript on behalf of all authors: Mummareddy. Statistical analysis: Mummareddy.

\section{Correspondence}

Nishit Mummareddy, Department of Neurological Surgery, Vanderbilt University Medical Center, 4906 Illinois Ave., Nashville, TN 37209. email: nishit.mummareddy@vanderbilt.edu. 